\title{
Effect of Body Mass Index on Lipid Profile of Type 2 Diabetic Patients at An Urban Tertiary Hospital In Nigeria
}

\author{
Funmilayo Esther Omotoye*, Grace Tanimoowo Fadupin \\ Department Of Human Nutrition, Faculty Of Public Health, University Of Ibadan, Ibadan, Oyo State, Nigeria.
}

\begin{abstract}
Diabetes mellitus is an important global public health problem. In addition to being a chronic disease, obesity is a key risk factor for Type 2 diabetes mellitus (T2DM) that leads to disability. This study aimed at investigating the effect of Body Mass Index on serum lipid profile in type II diabetic patients.

The study was descriptive cross sectional in design. Fifty consenting adults, aged 40-72years type II diabetic patients were studied. The socio-demographic characteristics were assessed through semi-structured interviewer administered questionnaire. Anthropometric indices of height and weight were assessed to determine body mass index (BMI). The serum lipid profile of participants for the last six months was assessed from their hospital records. The effect of the BMI on their lipid profile was determined using correlation coefficient and chi-square. Mean age of respondents was $57.82 \pm 3.3$ years. Out of the participants $36 \%$ had normal weight, while 64\% were either overweight or obese. The BMI of participants had an inverse relationship with HDL but positive relationship with LDL, total cholesterol and triglyceride levels of the participants. However, the effect of BMI was only significant on triglyceride level of respondents $(P=0.023)$. Normal BMI significantly improves dyslipidemia in type 2 diabetic patients.
\end{abstract}

Keywords: Type II diabetes, serum lipids profile, Body mass index, obesity, overweight.

Word count: 197

\section{Introduction}

Diabetes is associated with a greater risk of morbidity and mortality from cardiovascular disease (CVD), and heart disease is the leading cause of death among people with diabetes [1]. Diabetes mellitus is a chronic disease that requires long term medical attention both to limit the development of its devastating complications and manage them when they occur. It is more common amongst developed countries where affluent and overweight individuals live longer than in under developed countries [2].

Dyslipidaemia is a well recognised and modifiable risk factor for cardiovascular diseases which is currently a leading cause of morbidity and mortality world-wide [1]. Dyslipidemia is common in DM, as both insulin deficiency and resistance affects enzymes and pathways of lipid metabolism [3]. Diabetic dyslipidaemia is characterized by raised triglycerides, low high-density lipoprotein, raised apo-B, and small dense low density lipoprotein particles. It may be present at the diagnosis of type 2DM and it is a component of the metabolic syndrome. The pathogenesis of heart disease in diabetes is complex, but serum lipids are frequently abnormal and likely contribute to the risk of coronary artery disease [1]. Lipids and lipoproteins are well known risk factors for ischemic heart disease. Elevated levels of triglyceride, cholesterol, and LDL are documented as risk factors for atherogenesis. It is noteworthy that CRP plasma levels even slightly higher from the conventional upper limit of normal $(1 \mathrm{mg} / \mathrm{dL})$ have been associated with a 2-3-fold increase in risk of future myocardial infarction, stroke, and peripheral atherosclerosis among apparently healthy middle-aged men and women [4].

A worldwide epidemic exists with respect to diabetes mellitus, primarily because of increased rates of obesity. Obesity has become widespread in developed countries along with a corresponding increase in the prevalence of diabetes [5]. Epidemiological studies have shown that, compared to lean individuals, very obese men and women (body mass index >35) have several folds increase in probability of developing Type 2 diabetes [2]. It has been established that BMI is a significant predictor of cardiovascular disease and type 2 diabetes mellitus [6].

Obesity can be described as an imbalance between energy intake and expenditure such that excess energy is stored in fat cells, which enlarge or increase in number. Obesity is defined as a body mass index (BMI) of $>30 \mathrm{~kg} / \mathrm{m}^{2}$, according to WHO criteria. Obesity and overweight are significant public health problems worldwide, affecting an estimated 1 billion persons and contributing to hypertension, type 2 diabetes mellitus, cardiovascular disease, and death. Its prevalence in developed countries, such as the United States, is as high as $26.6 \%$ in men and $32.2 \%$ in women above age 20 years [3].

In this study, we aimed to examine the effect of BMI on lipid profile in type 2 diabetic patients attending university college hospital Ibadan, Oyo State, Nigeria. 


\subsection{Study location}

\section{Method}

This cross-sectional study was carried out at the university college hospital, Ibadan, Oyo State, Nigeria. A total of 50 type II diabetic patients (20 males and 30 females) attending the medical outpatient clinic (MOP) of the university college hospital (UCH), Ibadan, Oyo State, Nigeria from March to August 2006 were included in this study. All the type II diabetic patients who gave their consent and had their serum lipid profile assessed within the last six months was evaluated. Only those in attendance for two consecutive months took part in the study. Record of their lipid profile: low density lipoprotein (LDL), high density lipoprotein (HDL), Total cholesterol (TC) and Triglyceride (TG) were obtained from their hospital record.

\subsection{Data collection technique}

A semi structured interviewer administered questionnaire was used to interview each patient that gave their consent to participate in the study and also had his/her lipid profile assessed within the last six months. Information with regards to the participants' socio-economic data namely, age, sex, income, tribe, religion, occupation, and educational status were obtained with the aid of the questionnaire.

Record of their lipid profile level was obtained from their hospital records. The target lipid values were LDL <130mg/dl, HDL >40mg/dl, Triglyceride $<150 \mathrm{mg} / \mathrm{dl}$, and Total cholesterol <200mg/dl. Anthropometric indices of weight and height were obtained to derive body mass index. Weight was measured in kilograms with bathroom-weighing scale CAP.260 lbs $(120 \mathrm{~kg})$ GRAD.2 lb $(0 \mathrm{~kg})$ made in China by HANA-The big boss. Weight was taken with the participant in standing position, with light cloth and without shoes. Height was measured with a stadiometer manufactured and standardised by human Nutrition department of the university of Ibadan, Nigeria for research purposes. It was recorded in meters with the participants also in standing position, without shoes, cap or head gear. Body mass index was calculated as weight in kilograms per height in meters squared $\left(\mathrm{kg} / \mathrm{m}^{2}\right)$. A BMI of $<18.5 \mathrm{~kg} / \mathrm{m}^{2}$ was recorded as underweight, $18.5-24.9 \mathrm{~kg} / \mathrm{m}^{2}$ as normal weight, 25 $29.9 \mathrm{~kg} / \mathrm{m}^{2}$ as overweight and $>30 \mathrm{~kg} / \mathrm{m}^{2}$ as obesity [7].

\section{Definitions}

By using the guidelines of the Third Report of the National Cholesterol Education Program Expert Panel on Detection, Evaluation, and Treatment of High Blood Cholesterol in Adults (Adult Treatment Panel III) for serum lipid profile reference level, hypercholesterolemia was defined as TC $>200 \mathrm{mg} / \mathrm{dl}$, high LDL-C when value is $\geq 130 \mathrm{mg} / \mathrm{dl}$, hypertriglyceride as TG $>150 \mathrm{mg} / \mathrm{dl}$, and low HDL-C as $<40 \mathrm{mg} / \mathrm{dl}$. Dislipidemia was defined by presence of one or more abnormal serum lipid concentration. Definition for Normal TC was $<200 \mathrm{mg} / \mathrm{dl}$, LDL-C was $<130 \mathrm{mg} / \mathrm{dl}$, TG was $<150 \mathrm{mg} / \mathrm{dl}$ and HDL-C was $\geq 40 \mathrm{mg} / \mathrm{dl}$ [8].

The BMI is commonly used to classify individuals as underweight (less than $18.5 \mathrm{Kg} / \mathrm{m} 2)$, normal weight (18.5$24.9 \mathrm{Kg} / \mathrm{m} 2)$, overweight $(25.0-29.9 \mathrm{Kg} / \mathrm{m} 2)$, obesity class I $(30.0-34.9 \mathrm{Kg} / \mathrm{m} 2)$, class II $(35.0-39.9 \mathrm{Kg} / \mathrm{m} 2$ ) and class III (more than $40 \mathrm{Kg} / \mathrm{m} 2)[6,8]$.

\subsection{Data Analysis}

The data were evaluated by Statistical Package for Social Sciences (SPSS) 16.0 version software. Pearson's correlation test was performed to examine various correlations. Independent samples t-test (2-tailed) was used to compare means of different parameters between males and females. The results were considered statistically significant when $\mathrm{P} \leq 0.05$. The effect of body mass index on serum lipid profile was evaluated by one way analysis of variance (ANOVA).

\section{Result}

Table 1. Lipid profile, bmi and age of male and female type 2 diabetic patients

\begin{tabular}{|c|c|c|c|c|c|}
\hline \multirow[t]{2}{*}{ Parameters } & \multicolumn{2}{|l|}{ Male $(n=20)$} & \multicolumn{2}{|c|}{ Female $(n=30)$} & \multirow[b]{2}{*}{$\mathrm{p}$} \\
\hline & Mean \pm SD & Range & Mean \pm SD & Range & \\
\hline \multicolumn{6}{|l|}{ Serum lipid Profile (mmol/L) } \\
\hline Cholesterol & $155.30 \pm 61.9$ & $45-271$ & $175.20 \pm 46.1$ & $96-274$ & 0.514 \\
\hline Triglyceride & $94.50 \pm 44.4$ & $31-216$ & $96.74 \pm 37.5$ & $20-170$ & 0.544 \\
\hline HDL & $46.95 \pm 15.0$ & $15-72$ & $52.30 \pm 21.4$ & $18-105$ & 0.383 \\
\hline LDL & $111.95 \pm 41.9$ & $31-185$ & $110.50 \pm 41.9$ & 31.217 & 0.302 \\
\hline Body mass index $\left(\mathrm{Kg} / \mathrm{m}^{2}\right)$ & & & & & $0.042 *$ \\
\hline Mean $\pm \mathrm{SD}=28.90 \pm 11.9$ & $\mathrm{n}(\%)$ & & $\mathrm{n}(\%)$ & Total $\mathrm{n}(\%)$ & \\
\hline 18.5- 24.9 (Normal weight) & $11(55.0)$ & & $7(23.3)$ & $18(36.0)$ & \\
\hline $25.0-29.9$ (overweight) & $8(40.0)$ & & $10(33.3)$ & $5(10.0)$ & \\
\hline 30.0-34.9 (obesity class 1 ) & $1(5.0)$ & & $9(30.0)$ & $10(20.0)$ & \\
\hline 35.0-39.9(obesity class 2) & $0(0)$ & & $3(10.0)$ & $6(6.0)$ & \\
\hline$>40.0($ Extreme obesity class 3$)$ & $0(0)$ & & $1(3.3)$ & $1(2.0)$ & \\
\hline Age (Years) & & & & & $0.020 *$ \\
\hline Mean Age \pm SD & $60.85 \pm 8.0$ & & $55.80 \pm 8.57$ & $57.82 \pm 3.3$ & \\
\hline
\end{tabular}


$*$ = statistically significant

The result (Table 1) showed that none of the participant was underweight. Only $36.0 \%$ had normal weight while $64.0 \%$ was either overweight or obese. The difference in the body mass index of male and female respondents was significant $(\mathrm{p}=0.042)$. A total of $66.0 \%$ of the participants had abnormal high density lipoprotein values $(<60 \mathrm{mg} / \mathrm{dl})$ while $12.0 \%$ had border line or high triglyceride level. Also $30 \%$ had abnormal low density lipoprotein level while $34 \%$ had borderline or high total cholesterol level.

Table 2: Mean BMI Values $\left(\mathrm{kg} / \mathrm{m}^{2}\right)$ by age and sex

\begin{tabular}{|lccclll|}
\hline Age range (years) & \multicolumn{3}{c}{ Male } & \multicolumn{4}{c|}{ Female } \\
& No. BMI Mean \pm SD & SE & No. & BMI Mean \pm SD & SE \\
\hline $40-49$ & 2 & $21.82 \pm 2.0$ & 1.42 & 6 & $31.68 \pm 7.8$ & 3.20 \\
$50-59$ & 6 & $25.22 \pm 3.1$ & 1.25 & 17 & $28.02 \pm 4.3$ & 1.04 \\
$60-69$ & 8 & $25.59 \pm 3.3$ & 1.16 & 5 & $42.69 \pm 34.9$ & 15.62 \\
$70-79$ & 4 & $26.16 \pm 2.3$ & 1.14 & 2 & $30.48 \pm 6.5$ & 4.57 \\
Total sample & 20 & $25.21 \pm 2.9$ & 0.67 & 30 & $31.36 \pm 14.8$ & 2.70 \\
\hline Abbreviations: BMI, body mass index; SE, standard error, No., Number \\
\multicolumn{7}{l}{} \\
\hline
\end{tabular}

Table 3. Correlation between socio-demographic characteristics of participants and their fasting lipid profile

\begin{tabular}{|lcccc|}
\hline \multirow{2}{*}{ Variables } & FASTING LIPID PROFILE (r-values (p-values)) & \\
Hex & $0.08(0.38)$ & $-0.11(0.30)$ & $0.02(0.51)$ & $0.05(0.54)$ \\
Age & $-0.01(0.95)$ & $0.13(0.30)$ & $0.04(0.49)$ & $-0.14(0.64)$ \\
Tribe & $0.06(0.67)$ & $-0.05(0.74)$ & $0.09(0.54)$ & $-0.11(0.44)$ \\
Marital status & $-0.48(0.74)$ & $0.01(0.95)$ & $0.07(0.64)$ & $-0.09(0.55)$ \\
Religion & $0.27(0.05)$ & $-0.05(0.07)$ & $-0.04(0.77)$ & $-0.02(0.89)$ \\
Educational Attainment & $-0.08(0.34)$ & $0.11(0.60)$ & $0.14(0.85)$ & $0.30(0.31)$ \\
Occupation & $-0.19(0.63)$ & $0.07(0.42)$ & $-0.13(0.45)$ & $0.04(0.09)$ \\
Income & $-0.09(0.78)$ & $0.11(0.71)$ & $0.15(0.12)$ & $0.31(0.55)$ \\
\hline
\end{tabular}

As shown in Table 3, the age, marital status, highest educational attainment, occupation, and income of the participants had negative relationship with HDL while sex, tribe and religion had positive relationship with HDL though the relationship was not significant $(\mathrm{p}>0.05)$. Only religion and occupation had a negative relationship with total cholesterol.

Table 4. Association between body mass index and serum lipid profile

\begin{tabular}{|c|c|c|c|c|}
\hline Body mass index $\left(\mathrm{kg} / \mathrm{m}^{2}\right)$ & \multicolumn{3}{|c|}{ Lipid profile $($ mean \pm SD) } & \multirow[b]{2}{*}{ Total cholesterol } \\
\hline Triclyceride & HDL & LDL & & \\
\hline Normal weight $18.5-24.9$ & $42.78 \pm 17.6$ & $117.39 \pm 48.1$ & $168.89 \pm 64.9$ & $92.05 \pm 27.9$ \\
\hline $\begin{array}{l}\text { Overweight } 25.0-29.9 \\
103.67 \pm 44.6\end{array}$ & \multicolumn{2}{|c|}{$52.83 \pm 14.3$} & $107.56 \pm 34.8$ & $169.17 \pm 51.9$ \\
\hline Obesity class $130.0-34.9$ & $57.60 \pm 26.0$ & $108.10 \pm 47.8$ & $156.90 \pm 36.3$ & $90.31 \pm 45.7$ \\
\hline Obesity class $235.0-39.9$ & $50.67 \pm 67$ & $88.67 \pm 38.7$ & $167.00 \pm 59.8$ & $65.33 \pm 34.3$ \\
\hline Extreme obesity class $3(\geq 40.0)$ & 59.00 & 129.00 & 207.00 & 170.00 \\
\hline r-values & -0.264 & -0.019 & 0.069 & 0.303 \\
\hline p-values & 0.231 & 0.799 & 0.375 & $0.023 *$ \\
\hline
\end{tabular}

$*=$ statistically significant

Table 5. Correlation between lipid profile and body mass index in type ii diabetics

\begin{tabular}{|c|c|c|c|}
\hline \multirow[t]{2}{*}{ Lipid profile } & \multicolumn{3}{|c|}{ Body Mass Index $\left(\mathrm{kg} / \mathrm{m}^{2}\right)$ Pearson correlation (sig. 2 tailed) } \\
\hline & Normal weight $18.5-24.9$ & Overweight $25-29.9$ & Obesity $\geq 30$ \\
\hline HDL & $-0.162(0.52)$ & $0.369(0.13)$ & $0.552(0.04)^{*}$ \\
\hline LDL & $0.369(0.13)$ & $0.491(0.04)^{*}$ & $0.419(0.14)$ \\
\hline Total Cholesterol & $0.211(0.40)$ & $0.074(0.77)$ & $-0.451(0.11)$ \\
\hline Triglyceride & $0.047(0.85)$ & $0.150(0.55)$ & $-0.356(0.21)$ \\
\hline \multicolumn{4}{|l|}{$*=$ Significant } \\
\hline \multicolumn{4}{|c|}{$\begin{array}{l}\text { Among the males BMI positively correlated with HDL }(\mathrm{r}=0.033) \text {, LDL }(0.139) \text {, Total cholesterol }(0.014) \text {, and triglyceride } \\
(0.182) \text {. Among the females BMI positively correlate with HDL }(0.495) * \text { and LDL }(0.218) \text { but negatively correlate with Total } \\
\text { cholesterol }(-0.331) \text { and Triglyceride }(-0.322) \text {. }\end{array}$} \\
\hline
\end{tabular}




\section{Discussion}

Diabetes mellitus is often associated with cardiovascular morbidity and this may partly be explained by the abnormal lipid profile which is sometimes a feature of diabetes [9]. We studied the effect of body mass index on lipid profile of type 2 diabetic patients at a tertiary hospital in Nigeria. Many studies have shown an association between BMI and triglycerides, and the association between lipid profile and body fat distribution had been much discussed during the past decades [6]. Both lipid profile and body fat have been shown to be the important predictors for metabolic disturbances including dyslipidemia, hypertension, diabetes, cardiovascular diseases, hyperinsulinaemia etc. [6]. This study showed positive correlation between lipid profile and overweight/obesity. Obesity is said to predispose individuals to diabetes [9] while dyslipidemia is associated with both obesity and diabetes mellitus [10].

In this study, the mean TC, Triglyceride and HDL were higher among type 2 diabetic females than males but LDL was higher among males than females. This was similar to a study by [2] which showed that mean TC, Tg, LDL and HDL were higher among the type 2 diabetic females than males. The gender differences in Lipid profile were not significant in this study. This was contrary to the findings in a study which showed that there were significant differences in TG, HDL-C, and LDL-C concentrations between men and women, whereas the mean concentration of TC was similar in both genders $(P=0.083)$ [11]. It was also reported in the study that serum lipid concentrations were significantly positively (including: TC, TG, LDL-C) or negatively (HDL-C) correlated to diabetes, hypertension, body size, and age [11].

This study showed that mean BMI was higher among females than males in all the age groups and that the overall mean BMI was significantly higher in females than in males (31.37 vs. 25.21$) \mathrm{kg} / \mathrm{m} 2$. These results were higher than the previously published mean BMI of participants from an urban community in Yemen (23.9 (SD 5.1) $\mathrm{kg} / \mathrm{m} 2$ and. 21.8 (SD 8.9) $\mathrm{kg} / \mathrm{m} 2$ in females and males, respectively) [12] and also the work of [13] which showed that the overall mean BMI was significantly higher in females than in males (28 vs. 25.4). Among the males BMI was highest in the 70-79 years group while 60-69 years had the highest among the females. The result of this study was against the findings of [14] which showed that women had a significantly lower BMI ( $\mathrm{P}<0.001)$, lower WC ( $<<0.001)$ and lower TC: HDL cholesterol ( $<<0.001)$ compared to men.

In our study, patients with DM who were overweight or obese accounted for $64 \%$ of the total investigated population with DM. This figure was the same with that of a study by [13] but higher than the findings of a previous study in Yemen that overweight and obesity accounted only for $26.2 \%$ of patients with type 2 DM aged 20-65 [13]. The worldwide data Analysis concerning the association between BMI and both morbidity and mortality suggested that the association of BMI with most diseases was rather continuous [15] and generally, women had a higher mean BMI than men [16].

Socioeconomic status is a predisposing factor for diabetes mellitus morbidity[17]. This study revealed that Education and income had positive relationship with LDL-C, Total cholesterol and Triglyceride but have negative relationship with HDL-C. This was against the findings of a study by [18] which showed that low educational level had higher levels of LDL-cholesterol and triglycerides, among both men and women, educational level appeared to be the strongest correlate of lipid levels. Previous Canadian research has found lower educational achievement level to be associated with increased prevalence of Type 2 diabetes [19]. Another study showed that diabetes increased monotonically with lower education levels in females, whereas for males only those in the lowest educational strata were more likely to develop diabetes [20].

Some have reported higher cholesterol levels among those with higher education or higher-grade of employment while some found no association at all in some studies [18]. In developing countries the association is often positive with those in higher socio-economic status having higher levels of serum lipids. In most of these studies, socio-economic status was usually measured using only one indicator of socio-economic status, mainly educational level [18]. Sex and occupation were positively associated with plasma triglyceride levels of participants. These findings agree with the report of [21] which indicate that sex and occupation activities were positively associated with the plasma cholesterol of type II diabetics individuals in Kuwait.

Our result showed that age had positive relationship with LDL-C and TC but had negative relationship with HDL-C and triglyceride. This was against the findings of [22] that revealed that age correlated negatively with total cholesterol $(\mathrm{CC}=-0.322, \mathrm{P}=0.001)$ and LDL-cholesterol $(\mathrm{CC}=-0.352, \mathrm{P}=0.000)$. The elderly females tend to have significantly higher HDL-cholesterol compared with the elderly males. Both total cholesterol and LDL-cholesterol are likely to decrease with age in those above 55 years old. The result of another study [23] showed increase in total cholesterol, triglyceride and LDL-Cholesterol and decrease in HDL-Cholesterol in women in perimenopausal period as compared to women in the younger age group.

With regards to relationship between BMI and lipid profile this study showed in Table 4 that the entire BMI group had weak positive relationship with Total cholesterol and triglyceride while they have weak negative relationship with LDL and HDL. All the subjects had normal HDL they mostly fell within the normal range of LDL, Total cholesterol and triglyceride except the one in obesity class 3 who had high LDL and total cholesterol. Those in obesity class 2 and 3 had high triglyceride. Only the relationship with triglyceride was 
significant. This was similar to the findings of [14] which reported that both WC and BMI were significantly associated with TG and TC: HDL cholesterol.

This study also revealed that as BMI increases plasma LDL, total cholesterol, and triglyceride increases. These findings are similar to the report of [24] which showed that the prevalence of dyslipidemia increased with BMI in their study which was consistent with some previous studies. The lipid abnormalities in overweight and obesity is likely a consequence of insulin resistance [11]. The rapid development of overweight or obesity in Shenzhen will undoubtedly accelerate the prevalence of dyslipidemia [11]. Obesity increases cardiovascular risk through risk factors such as increased fasting plasma triglycerides, high LDL cholesterol, low HDL cholesterol, elevated blood glucose and insulin levels and high blood pressure. Novel lipid dependent, metabolic risk factors associated to obesity are the presence of the small dense LDL phenotype, postprandial hyperlipidemia with accumulation of atherogenic remnants and hepatic overproduction of apoB containing lipoproteins [25].

Risk factors associated with dyslipidemia were aging, higher body size, hypertension, diabetes, and smoking. Dyslipidemia is becoming an important public health problem. Obesity can be used to screen for dyslipidemia along with other coexisting risk factors such as hypertension and diabetes. There is an urgent need to institute more positive public health measures and screening programs and to treat patients diagnosed with dyslipidemia [11]

A systematic review suggests that a weight reduction of 5\%or $10 \%$ in obese patients has a simultaneous improvement in the serum lipid profile [26]. A study revealed that a moderate-fat weight-loss and weightmaintenance diet improves the cardiovascular disease risk profile on the basis of favourable changes in lipids and lipoproteins. The moderate-fat diet elicited favourable changes in the lipoprotein profile [27].

This study showed obese diabetic individuals have dyslipidemia and more prone to develop cardiovascular diseases. In a study obese type-2 diabetic patients when compared to obese control subjects showed statistically significant increase in the levels of serum total cholesterol ( $<<0.001)$, serum triglycerides $(\mathrm{p}<0.001)$, serum LDL-cholesterol $(\mathrm{p}<0.001)$ while serum HDL-cholesterol levels did not show statistically significant difference in the two group $(\mathrm{p}>0.05)$ [28]. Another study showed that obesity is permanently associated with unhealthy lipid profile characterized by high triglycerides and LDL-C, and low HDL-C [2].

This study revealed that overweight had positive correlation with HDL, LDL, total cholesterol and triglyceride. The relationship between overweight and LDL was significant. Obesity correlated positively with HDL and LDL while it had negative correlation with total cholesterol and triglyceride. This was in line with a finding which showed that BMI correlated positively with TC and LDL-C in Type 2diabetics [29]. Type 2 diabetes mellitus is characterized by dyslipidemia the level of which is associated with BMI [29]. The study by [29] also showed that Cholesterol levels in turn correlate with BMI indicating that lipid levels are influenced by BMI. A study showed that positive correlations were seen only between BMI $>25 \mathrm{~kg} / \mathrm{m}^{2}$ and VLDL in the total study group [30]. Plasma lipids were positively correlated with BMI and artherogenic indices, except for HDL$\mathrm{C}$, which was negatively correlated to artherogenic indices and LDL-C but was positively correlated with BMI in another study [31]. Our finding was also similar to that of a study which revealed a proportional correlation between serum triglyceride level and BMI, with the highest triglyceride levels observed in overweight and obese patients [2].

Both lipid profile and body fat have been shown to be the important predictors for metabolic disturbances including dyslipidaemia, hypertension, diabetes, cardiovascular diseases, hyperinsulinaemia. Association of lipid profiles is reported with Lifestyle, age, intra-abdominal adiposity, Obesity and BMI [6].

In our study, serum lipid concentrations showed stronger correlations in males than in females with BMI. This is similar with a study that revealed that serum lipid concentrations showed stronger correlations in males than in females not only with BMI but also with TBF [32].

\section{Conclusion}

Our results demonstrated that the mean BMI, as a continuous variable associated with lipid profile and was significantly higher in females than in males at different age groups. As BMI increases plasma LDL, total cholesterol, and triglyceride increases. Dislipidemia was higher among type 2 diabetic females than males. Obesity increases cardiovascular risk through risk factors such as increased fasting plasma triglycerides, high LDL cholesterol, low HDL cholesterol. Obese diabetic individuals have dyslipidemia and more prone to develop cardiovascular diseases. Obesity can be used to screen for dyslipidemia along with other coexisting risk factors such as diabetes.

\section{References}

[1]. JA Kayode, AO Sola, AS Matthew, BO Adesola, I Ademola, AT Adedeji and AS Adelani. Lipid profile of type 2 diabetic patients at a rural tertiary hospital in Nigeria. Journal of Diabetes and Endocrinology, 1(4), 2010, 46-51.

[2]. ZU Ali and MS Al-Zaidi. The Association Between Body Mass Index, Lipid Profile and Serum Estradiol Levels in a Sample of raqi Diabetic Premenopausal Women. Oman Medical Journal, 26(4), 2011, 263-266. 
[3]. L Gordon, D Ragoobirsingh, EYA Morrison, E Choo-Kang, D McGrowder, and E Martorell. Lipid Profile of Type 2 Diabetic and Hypertensive Patients in the Jamaican Population. J Lab Physicians, 2(1), 2010, 25-30.

[4]. H Rajkumar, N Mahmood, M Kumar, SR Varikuti, HR Challa, and SP Myakala. Effect of Probiotic (VSL\#3) and Omega-3 on ipid Profile, Insulin Sensitivity, Inflammatory Markers, and Gut Colonization in Overweight Adults: A Randomized, Controlled Trial. Hindawi Publishing Corporation Mediators of Inflammation, 2014, 1-8.

[5]. Riaz S, Alam SS, Raza M, Hasnain S, Akhtar MW. Obesity as risk factor and study of obesity related proteins in diabetes mellitus. Afr J Biotechnol 2009;8(5):737-744.

[6]. HS Sandhu, S Koley and KS Sandhu. A Study of Correlation between Lipid Profile and Body Mass Index (BMI) in Patients with Diabetes Mellitus. J. Hum. Ecol. 24(3), 2008, 227-229.

[7]. World Health Organization (WHO). Appropriate body-mass index for Asian populations and its implications for policy and ntervention strategies. The Lancet, 2004, 157-163.

[8]. Third Report of The National Cholesterol Education Program (NCEP) Expert Panel on Detection, Evaluation, and Treatment of High Blood Cholesterol in Adults (Adult Treatment Panel III). National Institutes of Health, 01-3670, 2001,3-18.

[9]. MA Devi, NS Singh, and SS Singh. Thyroid Dysfunction in Type 2 Diabetic Patients in Urban Area of Minipur. International Journal of Pharmaceutical Invention, 2, 2013, 7-9.

[10]. CEJ Udiong, MH Etukudoh, IK Isong, and EF Udoisa. Evaluation of BMI and Lipids Profile in Type 2 Diabetic Subjects with Low and Raised Levels of Thyroid Hormone in Calabar, Nigeria. Journal of Diabetes Mellitus, 5, 2015, $277-283$.

[11]. W Ni, X Liu, Z Zhuo, X Yuan, J Song, H Chi and J Xu. Serum lipids and associated factors of dyslipidemia in the adult population in Shenzhen. Lipids in Health and Disease, 2015, 14:71.

[12]. AA Gunaid . Obesity, overweight and underweight among adults in an urban community in Yemen. East Mediterr Health J. 18(12), 2012, 1187-93.

[13]. BA. Al-Sharafi, ${ }^{1, *}$ and AA. Gunaid. Prevalence of Obesity in Patients with Type 2 Diabetes Mellitus in Yemen. Int J Endocrinol Metab, 12(2), 2014, e13633.

[14]. DR Brenner, K Tepylo, KM Eny, LE Cahill and A El-Sohemy. Comparison of body mass index and waist circumference as predictors of cardiometabolic health in a population of young Canadian adults. Diabetology \& Metabolic Syndrome, 2, 2010:28.

[15]. MM Finucane, GA Stevens, MJ Cowan, G Danaei, JK Lin, CJ Paciorek, et al. National, regional, and global trends in body-mass index since 1980: systematic analysis of health examination surveys and epidemiological studies with 960 country-years and 9.1 million participants. Lancet, 377(9765), 2011, 557-67.

[16]. WPT James, R Jackson-Leach, CN Mhurchu, E Kalamara, M Shayeghi, NJ Rigby, et al. Overweight and obesity (high body mass index). In: Ezzati M, et al., editors. Comparative quantification of health risks: global nd regional burden of disease attributable to major risk factors. Geneva: World Health Organization, 2004, 497-596

[17]. G Veghari, M Sedaghat, H Joshaghani, SA Hoseini, F Niknezad, A Angizeh, E Tazik, P Moharloei. Association between sociodemographic factors and diabetes mellitus in the northof Iran: A population -based study. Science direct, 2(3), 2010, $154-157$.

[18]. S Shohaimi, MS Boekholdt, R Luben, NJ Wareham and K Khaw. Distribution of lipid parameters according to different socioeconomic indicators- the EPIC-Norfolk prospective population study. BMC Public Health, 14, $2014,782$.

[19]. K Dasgupta,S Khan, N Ross a. Type 2 diabetes in Canada: concentration of risk among most disadvantaged men but inverse social gradient across groups in women. Diabet Med [Internet], 27, 2010, 522-31.

[20]. LA. Rivera, M Lebenbaum, and LC. Rosella. The influence of socioeconomic status on future risk for developing Type 2 diabetes in the Canadian population between 2011 and 2022: differential associations by sex. Int J Equity Health, 14, $2015,101$.

[21]. F AI-Mahroos and K AI-Roomi. Diabetes in Arabian Gulf populations: an overview. Diabetes International Africa Edition 14(2), 2006,9-11

[22]. TA. Marhoum, AA. Abdrabo, MF. Lutfi. Effects of age and gender on serum lipid profile in over 55 years-old apparently healthy Sudanese individuals. Asian Journal of Biomedical and Pharmaceutical Sciences, 3(19), 2013, 10-14.

[23]. GI Deepti,S Sukanya, VR Ashalatha, A Sarfraz. Age related difference in the lipid profile in normal heal thy women. NUJHS, 4(2), 2014, 2249-7110

[24]. F Bayram, D Kocer, K Gundogan, A Kaya, O Demir, R Coskun, et al. Prevalence of dyslipidemia and associated risk factors in Turkish adults. J Clin Lipidol, 8(2),2014, 206-16.

[25]. B Klop, Jan WF Elte and MC Cabezas. Dyslipidemia in Obesity: Mechanisms and Potential Targets. Nutrients 5(4), 2013, 12181240 .

[26]. B Hutton and D Fergusson. Changes in body weight and serum lipid profile in obese patients treated with orlistat in addition to a hypocaloric diet: a systematic review of randomized clinical trials. American Journal of Clinical Nutrition, 80, 2004, $1461-8$.

[27]. CL Pelkman, VK Fishell, DH Maddox, TA Pearson, DT Mauger, and PM Kris-Etherton. Effects of moderate-fat (from monounsaturated fat) and low-fat weight-loss diets on the serum lipid profile in overweight and obese men and women. American Journal of Clinical Nutrition, 79, 2004, 204-12.

[28]. NK Yadav , C Thanpari , MK Shrewastwa , RK Mittal . Comparison of lipid profile in type-2 obese diabetics and obese nondiabetic individuals: a hospital based study from Western Nepal. Kathmandu Univ Med J (KUMJ), 10(39), 2012, 44-47.

[29]. CEJ Udiong, MH Etukudoh, IK Isong, EF Udoisa. Evaluation of BMI and Lipids Profile in Type 2 Diabetic Subjects with Low and Raised Levels of Thyroid Hormone in Calabar, Nigeria. Journal of Diabetes Mellitus, 5, 2015, 277-283

[30]. Y Himabindu, M Sriharibabu, K Alekhya, K Saisumanth, N Lakshmanrao, and K Komali. Correlations between anthropometry and lipid profile in type 2 diabetics. Indian J Endocrinol Metab, 17(4), 2013, 727-729.

[31]. EI Ugwuja, NC Ogbonna, AN Nwibo, IA Onimawo. Overweight and obesity, lipid profile and Artherogenic indices among civil servants in Abakaliki, south Eastern Nigeria. Annals of medical and Health sciences research, 1(1), 2013, 1-18

[32]. JW Choi, SH Pail and SK Kim. Associations Between Total Body Fat and Serum Lipid Concentrations in Obese Human Adolescents. Annals of Clinical \& Laboratory Science Summer, 32(3) 2002, 271-278. 